\title{
Lymphoid tissue inducer cells: bridges between the ancient innate and the modern adaptive immune systems
}

\author{
PJL Lane $^{1}$, FM McConnell ${ }^{1}$, D Withers ${ }^{1}$, F Gaspal $^{1}$, M Saini ${ }^{1}$ and G Anderson ${ }^{1}$
}

\begin{abstract}
Phylogeny indicates that adaptive immunity evolved first in diffusely distributed lymphoid tissues found in the lamina propria (LP) of the gut. B follicular structures appeared later, probably initially in isolated lymphoid follicles in the LP and then in organized lymphoid tissues such as lymph nodes and Peyer's patches. The development of these new lymphoid structures was enabled by gene duplication and evolution of new tumor necrosis family members. Here, we argue that lymphoid tissue inducer cells (LTis) had a pivotal role, not only in the development of organized lymphoid structures, but also in the subsequent genesis of the CD4-dependent class-switched memory antibody responses. In this review, we concentrate on the latter function: the sustenance by LTis of CD4 T-cell responses for protective immunity.
\end{abstract}

\section{INTRODUCTION}

Adaptive $\mathrm{T}$ - and $\mathrm{B}$-cell responses are a vertebrate invention. The main cellular players in immunity: CD4 and CD8 T cells, intraepithelial lymphocytes, B cells, and natural killer cells, appear to be present in all extant vertebrates, ${ }^{1}$ as are CD8 memory immune responses. ${ }^{2}$ The earliest vertebrates, the jawless agnathans, possessed pockets of primitive gut-associated lymphoid tissue, diffusely distributed throughout the lamina propria (LP). Along with the discrete lymphoid organs, thymus, and spleen, recombination-activating gene-dependent $T$ and B cells first appeared in jawed vertebrates, ${ }^{3}$ all of which show allogeneic graft rejection with functional evidence of accelerated recall responses (memory). ${ }^{4,5}$ With progression through the more recently evolved vertebrate phyla, a fundamental difference in immunological function emerges. Only mammals have proper encapsulated lymph nodes and other organized lymphoid structures such as Peyer's patches. The advantage conferred by their acquisition is the capacity to make exquisitely specific high-affinity class-switched antibody responses, a function which is strikingly absent in lower vertebrates. ${ }^{6}$ Crucial to this immunity are CD4 $\mathrm{T}$ cells, which exert their effects by interacting with B cells. In their naïve state, and unlike CD8 T cells, CD4 $\mathrm{T}$ cells require secondary lymphoid tissues for their survival in mice. ${ }^{7}$ It is noteworthy that CD4 memory T cells can survive independently of secondary lymphoid tissues, for example, in the $\mathrm{LP}^{8}$ and the bone marrow. ${ }^{9}$
We have argued that CD4 memory and lymphoid tissue organization co-evolved, the selected outcome being the capacity to make high-affinity memory antibody responses, and that lymphoid tissue inducer cells (LTis) are the fundamental cellular components in this process. ${ }^{10}$ Recent data have indicated that this cell type secretes large amounts of the cytokine, interleukin 22 (IL22), ${ }^{11}$ which has been strongly linked with the promotion of integrity at epithelial surfaces. ${ }^{12}$ We suggest that the original presence of LTis, located in murine cryptopatches, was as a cellular component of the innate immune system occurring in the LP. However, as the immune system evolved, so did the function of LTis. Through the acquisition and expression of newly evolving tumor necrosis factor (TNF) family members, we propose that LTis provided for the development and organization of lymphoid structures, which could foster first high affinity and then memory CD4 responses. In this review, we try and identify the steppingstones that might link the development of ancestral LTis to their pivotal position in the adaptive CD4 immune response.

\section{LTis ANDTHE DEVELOPMENT OF ORGANIZED LYMPHOID STRUCTURES}

The novel $\mathrm{CD} 4^{+}$cell, now identified as the LTi, was first characterized in developing murine lymph nodes by Mebius and Weissman. ${ }^{13}$ The development of these cells was subsequently shown to depend on the splice variant of the transcription factor,

${ }_{1}^{1}$ MRC Centre for Immune Regulation, Institute for Biomedical Research, Birmingham Medical School, Vincent Drive, Birmingham, UK. Correspondence: PJL Lane (p.j.l.lane@bham.ac.uk)

Received 12 June 2009; accepted 10 August 2009; published online 9 September 2009. doi:10.1038/mi.2009.111 
retinoic acid orphan receptor- $\gamma(\mathrm{ROR} \gamma \mathrm{t}) .{ }^{14-16}$ Their role in the induction of both conventional ${ }^{17,18}$ and inducible ${ }^{19}$ lymphoid tissues has been reviewed elsewhere. ROR $\gamma \mathrm{t}^{+}$LTis in mice are characterized by their expression of CD4 and interleukin 7 receptor- $\alpha$ in the absence of lineage markers such as CD3, B220, or CD11c. ${ }^{17}$ Human LTis appear to lack CD4, but otherwise share a very similar phenotype to their murine equivalents. ${ }^{20}$

Lymphoid tissue inducer cells are not essential for the development of some lymphoid tissues, such as spleen, nasal-associated lymphoid tissues, ${ }^{21}$ or primitive aggregates of lymphocytes in the omentum, ${ }^{22}$ but are required for the development of conventional lymph nodes and also isolated lymphoid follicles (ILFs), which develop from crypopatches in large and small intestinal LP. Conventional ROR $\gamma \mathrm{t}^{+}$LTis are not essential for the formation of white pulp areas of the spleen, ${ }^{14,23}$ but they are present in the fetal spleen, ${ }^{24,25}$ in which they attach to and signal fixed stromal cells. These stromal cells respond by expressing homeostatic chemokines, which guide the recruitment of $\mathrm{T}$ cells, dendritic cells, and B cells to form the white pulp microenvironments that support CD4 T-cell responses.

Although originally only thought to be functional in the embryo, LTis are also present in adult life. In adult mice, LTis have been implicated in the induction of de novo ILFs in the gut $^{19}$ and in the repair of secondary lymphoid tissues, ${ }^{26}$ a function analogous to the one they perform in fetal life.

It is to be noted that single cell PCR analysis shows that both murine fetal and adult splenic LTis are heterogeneous and consist of three distinct populations on the basis of expression of the homeostatic chemokine receptors, chemokine (C-X-C motif) receptor 5 (CXCR5) and chemokine (C-C motif) receptor (CCR7). ${ }^{27}$ This heterogeneity of chemokine receptor expression is consistent with the notion that distinct LTi populations reside within distinct locations in the adult murine spleen, for example, associated with B follicle stromal cells (CXCR $\left.5^{+} \mathrm{CCR}^{-}{ }^{-} \mathrm{LTi}\right)$, at the boundary between the $\mathrm{B}$ and $\mathrm{T}$ cell areas $\left(\mathrm{CXCR} 5^{+} \mathrm{CCR} 7^{+}\right.$ $\mathrm{LTi}){ }^{28}$ and in the central T-zone areas adjacent to T-zone stroma (CXCR5 ${ }^{-}$CCR7 ${ }^{+}$LTi). ${ }^{29}$

\section{Signals to CD4 T cells}

In the T-zone, LTis interact with the underlying stroma, dendritic cells, and T cells. ${ }^{29}$ Associations between primed CD4 $\mathrm{T}$ cells and LTis in B follicles and at the B/T interface are also apparent. ${ }^{28}$ The latter point is consistent with provision of signals from LTi to CD4 T cells.

\section{LTis EXPRESS RECENTLY EVOLVEDTNF FAMILY MEMBER LIGANDS}

The TNF family members of receptors and ligands are closely associated with the development and organization of adaptive immune responses. Many members of this TNF family occur in a cluster found in all vertebrates, ${ }^{30}$ although not all TNF genes are conserved. Examples of receptor-ligand interactions conserved throughout the vertebrate phylum are: $\mathrm{CD} 40$-ligand (TNFSF5, expressed on T cells), through its interaction with CD40 (TNFRSF5, expressed on B cells), has the pivotal role in $\mathrm{T}$ cell help for $\mathrm{B}$ cells ${ }^{31,32}$ and $\mathrm{B}$ cell activating factor (BAFF) and a proliferation-inducing ligand (APRIL) are TNF ligands implicated in $\mathrm{B}^{-33}$ and plasma cell ${ }^{34,35}$ survival, respectively.

Local gene duplication is a key source of variation between individuals. ${ }^{36}$ Iterated over millions of generations, as species diverge, diversification of duplicated genes provides the genetic fuel for the acquisition of new genes that control new functions. Comparison of fish and mammalian TNF family members identifies six new genes, ${ }^{30}$ namely, the lymphotoxin genes (LT $\alpha$ and LT $\beta$ ), OX40-ligand (OX40L, and TNFSF4), GITRL (TNFSF18), CD30L (TNFSF8), and CD27L (TNFSF7).

The receptors for these genes are located in two TNF clusters at the tips of human chromosomes 1 and 12 (Table 1). In addition to TNF receptor family members, the two gene clusters each contain a closely related serine protease, MASP2 (chromosome 1) and C1rs (chromosome 12), which catalyze the activation of complement component 4 through the mannan-binding pathway and the classical pathway, respectively. It seems likely that the two TNF clusters may have arisen from an ancestral chromosomal duplication that predated the evolution of jawed vertebrates, as originally proposed by Ohno. ${ }^{37}$ In support of this, although teleosts lack some TNF members, both gene clusters are present in fish genomes (www.ensembl.org).

Lymphoid tissue inducer cells are characterized by their expression of many different TNF ligands (TNFLs). However, they do not express any of the TNF members linked with B-cell activation and survival (CD40, BAFF, and APRIL). ${ }^{38}$ The expressed molecules not only include TNFLs shared with primitive vertebrates (TNF $\alpha$ (TNFSF2), LIGHT (TNFSF14), and TRANCE (TNFSF11A)) but also the more recently evolved TNFLs. Adult and embryonic LTis both express high levels of the lymphotoxins, LT $\alpha$ (TNFSF1) and LT $\beta$ (TNFSF3), and can be induced to express high levels of OX40L and CD30L. ${ }^{38}$

\section{HIGH-AFFINITY CLASS-SWITCHED ANTIBODIES DEPEND ON LT $\alpha$ - AND LT $\beta$-INDUCED ORGANIZATION}

Lymphotoxin- $\alpha$ and LT $\beta$, by binding to the TNF receptor, TNFRSF1A, and the lymphotoxin- $\beta$ receptor, provide key signals for the development of organized secondary lymphoid tissues, ${ }^{39}$ and the expression of LT $\alpha$ and LT $\beta$ on B cells is particularly important for the induction of CXCL13 and for B cell follicle formation. Signals through CD30 have additional roles in lymphoid tissue organization, ${ }^{40,41}$ and in the absence of lymphotoxin signals, a residual function for CD30 signals in the induction of the T-zone chemokine, CCL21, can be shown. ${ }^{41}$

The formation of discrete B-cell follicles enables CD4 T cells to orchestrate the $\mathrm{CD} 40$-dependent proliferation, somatic mutation, and selection of B cells within germinal centers (GCs), with the consequent production of high-affinity class-switched antibody responses. Lymphotoxin-deficient mice lack GCs and can produce neither high-affinity IgG in the systemic circulation ${ }^{42}$ nor IgA at mucosal surfaces. ${ }^{43}$ In contrast, low-affinity nonswitched IgM responses do not depend on organized lymphoid structures.

In mice in which the lymphocyte compartment of the spleen is disorganized (LT $\alpha^{-1-}, \mathrm{LT} \beta^{-/-}$, or $\mathrm{LT} \beta \mathrm{R}^{-/-}$), antibody responses are comparable with those in lower vertebrates. 
Table 1 Human TNF receptors on chromosomes 1 and 12

\begin{tabular}{|c|c|c|c|}
\hline TNF cluster 1 & $\begin{array}{c}\text { Chromosome } \\
1 \text { position } \\
\text { (Mb) }\end{array}$ & TNF cluster 2 & $\begin{array}{c}\text { Chromosome } \\
12 \text { position } \\
\text { (Mb) }\end{array}$ \\
\hline GITR & 1.13 & $T_{N F R 1^{\star}}$ & $12: 6.3$ \\
\hline $0 \times 40^{\star \star}$ & 1.14 & $L T \beta R^{\star \star}$ & $12: 6.36$ \\
\hline$H V E M^{\star \star \star}$ & 2.5 & $C D 27$ & $12: 6.4$ \\
\hline$D R 3^{\star}$ & 6.4 & $C D 4$ & $12: 6.7$ \\
\hline $4-1 B B$ & 7.9 & C1rs & $12: 7.1$ \\
\hline MASP2 & 11 & $A I D$ & $12: 8.6$ \\
\hline$C D 30$ & 12 & & \\
\hline TNFR2 $^{\star \star *}$ & 12.1 & & \\
\hline
\end{tabular}

Gene shown in bold italics show TNF receptors that are present in all vertebrates. Genes shown in italics show receptors whose ligands are only present in higher vertebrates. Genes shown in bold show the two serine proteases that catalytically cleave complement component, C4. Asterisked TNF receptors are close paralogues. Data obtained from www.ensembl.org.

Despite having the activation-induced cytidine deaminase (AID) that is required for both class switching and somatic mutation, ${ }^{44}$ intact T cell help through CD $40,{ }^{45}$ and somatic mutation of immunoglobulin genes, ${ }^{46}$ high-affinity antibody responses are not generated. This shows the key importance of establishing segregated B and T-cell areas for the generation of high-affinity antibodies. The implication is that the evolutionary drive for lymphoid organization was the acquisition of a mechanism ensuring the efficient selection of those B cells secreting highaffinity class-switched antibodies from the pool of candidates with mutated B-cell receptors.

The selective advantage conferred by high-affinity antibodies, in combination with the investment involved in their production, must have applied potent selective pressure for mechanisms to maintain them, now evolved as B and T-cell memory. Under normal circumstances, memory B and $\mathrm{T}$ cells collaborate with each other in the outer T-zone of secondary lymphoid structures. ${ }^{47}$ Organization is required for this interaction, as memory $\mathrm{B}$ and $\mathrm{T}$ cells fail to find each other in the absence of organization. ${ }^{48}$ This implies that lymphoid tissue organization was a prerequisite for the development of memory antibody responses.

\section{T-INDEPENDENT IgA PRODUCTION IN ILFS: A STEPTOWARD CD4-DRIVEN MEMORY ANTIBODY RESPONSES}

Recently, an intriguing insight into the evolution of CD4dependent memory class-switched antibody responses was revealed by the observation that LTis in ILFs are required for AID-dependent but T-independent IgA class switching in the gut. ${ }^{49}$ Birds and mammals share IgA but not IgG homologs, ${ }^{50}$ suggesting that IgA class switching evolved first in their common ancestor. The inability to switch to IgA in AID-deficient mice is associated with changes in gut commensal bacteria, ${ }^{51}$ and it has been shown that impaired production of IgA promotes inflammatory responses. ${ }^{52}$ ILFs develop postnatally, driven by bacterial colonization of the gut by commensal bacteria, ${ }^{53}$ suggesting that the presence of IgA prevents inappropriate inflammatory responses to beneficial bacteria.
In contrast to $\mathrm{T}$-dependent class switching, $\mathrm{T}$-independent IgA switching in ILFs depends on the TNFLs, BAFF and APRIL, provided by $\mathrm{CD} 11 \mathrm{c}^{+}$cells. ${ }^{49}$ Although LTis do not provide the BAFF and APRIL signals directly, LTis are required for the formation of ILFs, and are therefore essential for the formation of the cellular environment for IgA class switching and plasma cell formation.

The AID-dependent IgA class switching in ILFs seems to be a mechanism to generate plasma cells that produce a lessinflammatory polymeric immunoglobulin isotype. In contrast, T-cell-dependent CD40-driven GCs in conventional B follicles are specifically directed at production of high-affinity antibodies through AID-driven somatic hypermutation in GC B cells. Although GC B cells do undergo AID-dependent immunoglobulin class switching, terminal differentiation to plasma cells is blocked and $\mathrm{CD} 11 \mathrm{c}^{+}$cells, an integral component of ILFs, are excluded from conventional B follicles. Instead, the CD11 ${ }^{+}$ cells are found in the T-zone and at the $\mathrm{B} / \mathrm{T}$ interface, where they are also associated with LTis. Here, as in ILFs, AID expression is the route to class switching, and $\mathrm{CD} 11 \mathrm{c}^{+}$cells provide the BAFF and APRIL signals for plasma cell differentiation.

Figure 1 illustrates the potential evolution of ILFs from structures in which the LTis orchestrated class switching, depending on BAFF and APRIL from CD11 $\mathrm{c}^{+}$cells but not on T cells, to structures in which $\mathrm{T}$ cells were involved in $\mathrm{B}$-cell selection. Putatively, the next stage is the segregation of $\mathrm{CD} 11 \mathrm{c}^{+}$- and CD4 T-cell-dependent class switching into $\mathrm{B}$ follicles, primarily for the iterative CD4 T-cell selection of high-affinity B cells, and into the outer T-zone for the formation of immunoglobulin class-switched plasma cells.

\section{LACK OF CD4 MEMORY IN MICE DEFICIENT IN OX40 AND CD30}

The receptors for OX40 and CD30 are primarily expressed on activated T cells. ${ }^{54,55}$ Owing to our evidence that primed CD4 T cells could interact directly with LTis ${ }^{28}$ and that LTis readily express high levels of the ligands for CD30 and OX40, we grew mice deficient in OX40, CD30, or both molecules. Individual contributions of OX40 and CD30 signals to CD4 T-cell memory were identified, ${ }^{28,56}$ but the striking result was that when both signaling pathways were removed, CD4 memory generation was abrogated.

The first CD4 T-cell function we studied was help for B cells. There was no effect on primary immune responses, and in both in vitro and in vivo studies, CD4 T cells deficient in OX40 and CD30 initially proliferated quite normally. ${ }^{56}$ Therefore, there does not seem to be a requirement for OX40 and CD30 for the primary generation of antigen-specific $\mathrm{CD} 4 \mathrm{~T}$ cells or their initial expansion, just as primitive vertebrates lacking these genes exhibit normal primary immune responses. The impaired memory responses were not due to lack of appropriate positive selection in the thymus, as T-cell-receptor transgenic CD4 T cells deficient in OX40 and CD30 were positively selected, as they are normally. The key defect caused by the double deficit is in the capacity of primed CD4 T cells to survive, and OX40 and CD30 were found to have synergistic effects. The premature 


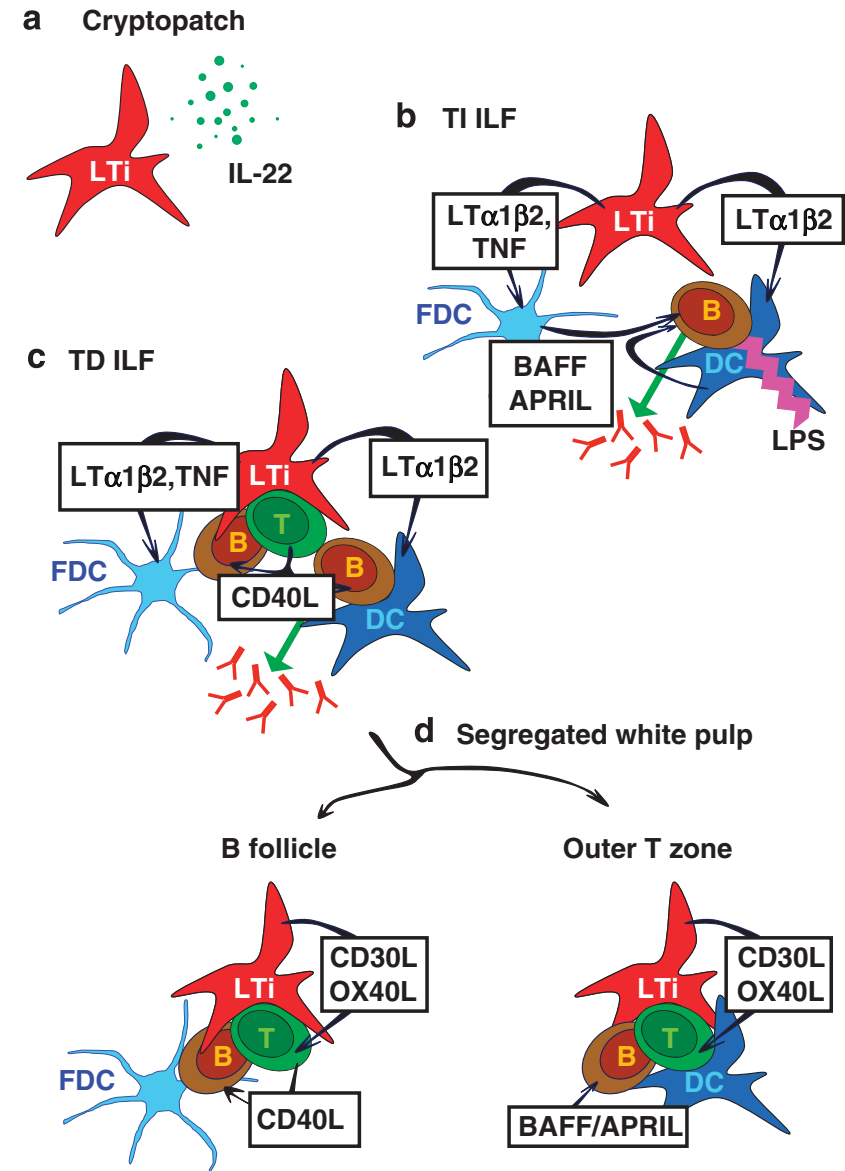

Figure $1 \mathrm{~A}$ putative model for role of LTis in innate and adaptive immunity. FDC, follicular dendritic cell, DC, dendritic cell, B, B cell, T, T cell, LTi, lymphoid tissue inducer cell. (a) Putative LTi-like cells, present within the primitive immune system of the gut, promote epithelial integrity at mucosal surfaces through secretion of IL22. (b) LTi, adapted through expression of the lymphotoxin genes, orchestrate the organization of $B$ cells and $\mathrm{CD}_{11 \mathrm{C}^{+}} \mathrm{DC}$ s into isolated lymphoid follicles (ILFs). These foster T-independent (TI) IgA class-switched plasma cells, mediated by BAFF and APRIL (expressed by DC), again to promote mucosal integrity. (c) Evolution of CD40-dependent T-cell selection within ILFs. LTis acquire the new TNF ligands (CD30L and OX40L), which evolve to promote CD4 memory. ${ }^{69}$ (d) Classical T-dependent (TD) responses in lymph node and spleen. In B follicles, CD40L-expressing CD4 T cells, LTis, and FDCs foster activation-induced cytidine deaminase (AID)-dependent affinity maturation in germinal center (GC). In the outer T-zone, $T$ (CD40L) and CD11 $\mathrm{c}^{+}$cells (BAFF and APRIL) foster AID-dependent class switching in $B$ cells that become plasma cells.

death of primed CD4 T cells was reflected in the CD4 T-cell driven GC response. GCs were initiated normally but terminated prematurely with the consequence that affinity maturation of the antibody response was grossly impaired. However, the most dramatic effect was on recall memory immune responses, which were vestigial in mice deficient in OX40 and CD30.

\section{MEMORY}

Our studies on double-deficient mice led us to conclude that OX40 and CD30 signals from LTis were critical for CD4 T-cell survival and memory. The importance of these molecules for CD4 survival extended beyond antibody responses, as mice deficient in signals through them were unable to sustain the CD4 Th1 response required to clear intracellular bacteria, ${ }^{57}$ suggesting that the mechanism for the induction of CD4 memory for antibody responses was shared with other CD4 T-cell effector/memory cells.

\section{LTis ANDTHE INDUCTION AND MAINTENANCE OF TOLERANCE}

In addition to their importance in the generation of functional secondary lymphoid tissues, we have shown that LTis are present in murine thymus, in which they are located adjacent to thymic medullary epithelium. ${ }^{58}$ Interestingly, there are similarities between the thymic medulla and the splenic T-zone, including common cellular components (mature T-cells, dendritic cells, and now LTis) and sites of CCR7-mediated lymphocyte migration. ${ }^{59}$ We have linked LTis expression of the TNFL, TRANCE (TNFSF11), with the generation of the first cohort of autoimmune regulator (AIRE)-expressing medullary thymic epithelial cells (mTEC) in the embryonic thymus, which enables intrathymic expression of tissue-restricted antigens for self-tolerance. Although positively selected thymocytes have also been shown to induce AIRE-expressing medullary thymic epithelial cells, ${ }^{60}$ early expression of AIRE is necessary and sufficient to prevent autoimmunity. ${ }^{61}$ Therefore, the contribution made by LTis in the development of $\mathrm{AIRE}^{+}$medullary thymic epithelial cells is an important component of self-tolerance.

As LTis are linked with the induction of tolerance to self and the generation of adaptive memory immune responses, we suggest that this is relevant to the mechanisms underlying autoimmunity. Self-tolerance is a cornerstone of adaptive immunity, ${ }^{62}$ and is particularly relevant in the context of CD4 memory, in which autoimmunity becomes a significant risk. The presence of both TRANCE and AIRE in early vertebrate genomes (www. ensembl.org) is consistent with AIRE-dependent mechanisms evolving early.

\section{PUTATIVE ORIGIN OF LTIS AND SUMMARY}

The key role of LTis in vertebrates in the development of lymphotoxin-dependent organization of lymph nodes and OX40 and CD30-dependent memory would place these cells late in the evolution of the vertebrate immune system. However, LTis express many genes that are expressed in all vertebrates, including IL22, ${ }^{11}$ which is strongly implicated in mucosal epithelial immunity. ${ }^{63,64}$ Recently, a population of LTi-like cells expressing high levels of IL22 has been described in the LP and gut-associated lymphoid tissue. ${ }^{65-67}$ In mice and men, these cells express ROR $\gamma$ t and also some natural killer markers, notably NKp46, ${ }^{65,67}$ although these cells lack natural killer function (cytotoxicity and interferon- $\gamma$ expression). These cells were located not only in the $\mathrm{LP}^{65,66}$ but also within murine cryptopatches. ${ }^{67}$ This has raised the possibility that LTis evolved first as innate cells maintaining epithelial integrity in the LP through expression of IL22 (Figure 1a). Recent data shows extensive synteny between the mammalian and the fish IL22 genetic loci, suggesting that this cytokine cluster was established in a common ancestor of mammals and fish. ${ }^{68}$ Murine cryptopatches, as their name suggests, 
are located between crypts at the base of the villi, and ROR $\gamma$ tdependent LTis are required for their formation, ${ }^{16}$ hence they are well placed to provide IL22 signals to the local intestinal epithelium (Figure 1a). Although cryptopatches have not been identified in other mammals, all mammals have ILFs; hence it is likely that LTis are also located adjacent to intestinal crypts in which the ILFs are formed.

Both IL22 and IgA promote mucosal defences, but the former is innate whereas the latter is part of the adaptive immune system. The evidence that cryptopatches became a focus for T-cellindependent B-cell selection and class switching to IgA through recruitment of $\mathrm{CD}_{11 c^{+}}$cells expressing BAFF and APRIL ${ }^{49}$ defines a transition in LTi function, linking them with both innate and adaptive immunity (Figure 1b). These new functions were dependent on the acquisition by LTis of the new TNF genes encoding the lymphotoxins. LTis expression of lymphotoxins initially enabled cryptopatches to recruit B cells and form ILFs, structures for primitive T-independent AID-dependent class switching. These structures subsequently evolved into T-dependent ILFs, supporting CD4 and CD40-dependent B-cell selection (Figure 1c). In conventional lymphoid tissues, the process of T-dependent AID-dependent selection within GCs is segregated from AID-dependent class switching to plasma cells associated with CD11c ${ }^{+}$dendritic cells (Figure 1d). Given the advantage against pathogens conferred by high-affinity memory antibody responses, it is likely that LTis, already keeping other cell types alive through TNFL expression, were the right niche for the expression of the late-evolving TNFLs, CD30L and OX40L, which supported survival of primed CD4 T cells. ${ }^{69}$

\section{ACKNOWLEDGMENTS}

This work was supported by a Wellcome Trust Programme grant to PL and GA.

\section{DISCLOSURE}

The authors declare no conflict of interest.

() 2009 Society for Mucosal Immunology

\section{REFERENCES}

1. Bernard, D. et al. Phenotypic and functional similarity of gut intraepithelial and systemic T cells in a teleost fish. J. Immunol. 176, 3942-3949 (2006).

2. Morales, H.D. \& Robert, J. Characterization of primary and memory CD8 T-cell responses against ranavirus (FV3) in Xenopus laevis. J. Virol. 81, 2240-2248 (2007).

3. Du Pasquier, L. Evolution of the immune system. In Fundamental Immunology (Paul, W.E., ed) 199-234 (Raven Press, New York, 1993)

4. Burnet, F.M. Evolution of the immune process in vertebrates. Nature 218, 426-430 (1968).

5. Haas, R. Transplantation reactions in the African lungfish, Protopterus amphibius. Transplantation 33, 249-253 (1982).

6. Zapata, A. \& Ameimiya, C.T. Phylogeny of lower vertebrates and their immunological structures. In Origin and Evolution of the Vertebrate Immune System (du Pasquier, L. and Litman, G.W., eds) 67-110 (Springer, Berlin, 2000).

7. Dai, Z. \& Lakkis, F.G. Cutting edge: secondary lymphoid organs are essential for maintaining the CD4, but not CD8, naive T cell pool. J. Immunol. 167, 6711-6715 (2001).

8. Reinhardt, R.L., Khoruts, A., Merica, R., Zell, T. \& Jenkins, M.K. Visualizing the generation of memory CD4 T cells in the whole body. Nature 410, 101-105 (2001).

9. Tokoyoda, K. et al. Professional memory CD4(+) T lymphocytes preferentially reside and rest in the bone marrow. Immunity 30, 721-730 (2009).
10. Lane, P.J.L., Gaspal, M.C. \& Kim, M.-Y. Two sides of a cellular coin: CD4+CD3 - cells orchestrate memory antibody responses and lymph node organisation. Nat. Rev. Immunol. 5, 655-660 (2005).

11. Takatori, H. et al. Lymphoid tissue inducer-like cells are an innate source of IL-17 and IL-22. J. Exp. Med. 206, 35-41 (2009).

12. Wolk, K. et al. IL-22 increases the innate immunity of tissues. Immunity 21, 241-254 (2004).

13. Mebius, R.E., Rennert, P. \& Weissman, I.L. Developing lymph nodes collect CD4+CD3 - LTbeta+ cells that can differentiate to APC, NK cells, and follicular cells but not T or B cells. Immunity 7, 493-504 (1997).

14. Sun, Z. et al. Requirement for RORgamma in thymocyte survival and lymphoid organ development. Science 288, 2369-2373 (2000).

15. Eberl, G. et al. An essential function for the nuclear receptor RORgamma(t) in the generation of fetal lymphoid tissue inducer cells. Nat. Immunol. 5, 64-73 (2004).

16. Eberl, G. \& Littman, D.R. Thymic origin of intestinal alphabeta T cells revealed by fate mapping of RORgammat+ cells. Science 305, 248-251 (2004).

17. Mebius, R.E. Organogenesis of lymphoid tissues. Nat. Rev. Immunol. 3, 292-303 (2003)

18. Eberl, G. \& Littman, D.R. The role of the nuclear hormone receptor RORgammat in the development of lymph nodes and Peyer's patches. Immunol. Rev. 195, 81-90 (2003).

19. Eberl, G. Opinion: inducible lymphoid tissues in the adult gut: recapitulation of a fetal developmental pathway? Nat. Rev. Immunol. 5, 413-420 (2005).

20. Cupedo, T. et al. Human fetal lymphoid tissue-inducer cells are interleukin 17-producing precursors to RORC+CD127+ natural killer-like cells. Nat. Immunol. 10, 66-74 (2009).

21. Harmsen, A. et al. Cutting edge: organogenesis of nasal-associated lymphoid tissue (NALT) occurs independently of lymphotoxin-alpha (LT alpha) and retinoic acid receptor-related orphan receptor-gamma, but the organization of NALT is LT alpha dependent. J. Immunol. 168, 986-990 (2002).

22. Rangel-Moreno, J. et al. Omental milky spots develop in the absence of lymphoid tissue-inducer cells and support $B$ and $T$ cell responses to peritoneal antigens. Immunity 30, 731-743 (2009).

23. Kurebayashi, S. et al. Retinoid-related orphan receptor gamma (RORgamma) is essential for lymphoid organogenesis and controls apoptosis during thymopoiesis. Proc. Natl. Acad. Sci. USA 97, 10132-10137 (2000).

24. Withers, D.R. et al. The role of lymphoid tissue inducer cells in splenic white pulp development. Eur. J. Immunol. 37, 3240-3245 (2007).

25. Vondenhoff, M.F. et al. Separation of splenic red and white pulp occurs before birth in a LTalphabeta-independent manner. J. Leukoc. Biol. 84, 152-161 (2008).

26. Scandella, E. et al. Restoration of lymphoid organ integrity through the interaction of lymphoid tissue-inducer cells with stroma of the T cell zone. Nat. Immunol. 9, 667-675 (2008).

27. Kim, M.Y. et al. Heterogeneity of lymphoid tissue inducer cell populations present in embryonic and adult mouse lymphoid tissues. Immunology 124, 166-174 (2008).

28. Kim, M.Y. et al. CD4(+)CD3(-) accessory cells costimulate primed CD4 T cells through $\mathrm{OX} 40$ and $\mathrm{CD} 30$ at sites where $T$ cells collaborate with $\mathrm{B}$ cells. Immunity 18, 643-654 (2003).

29. Kim, M.Y. et al. Function of CD4+CD3 - cells in relation to B- and T-zone stroma in spleen. Blood 109, 1602-1610 (2007).

30. Glenney, G.W. \& Wiens, G.D. Early diversification of the TNF superfamily in teleosts: genomic characterization and expression analysis. J. Immunol. 178, 7955-7973 (2007).

31. Banchereau, J. et al. The CD40 antigen and its ligand. Annu. Rev. Immunol. 12, 881-922 (1994).

32. Grewal, I.S. \& Flavell, R.A. A central role of $\mathrm{cd} 40$ ligand in the regulation of CD4(+) t-cell responses. Immunol. Today 17, 410-414 (1996).

33. Mackay, F. \& Browning, J.L. BAFF: a fundamental survival factor for B cells. Nat. Rev. Immunol. 2, 465-475 (2002).

34. Benson, M.J. et al. Cutting edge: the dependence of plasma cells and independence of memory B cells on BAFF and APRIL. J. Immunol. 180, 3655-3659 (2008).

35. Belnoue, E. et al. APRIL is critical for plasmablast survival in the bone marrow and poorly expressed by early-life bone marrow stromal cells. Blood 111, 2755-2764 (2008). 
36. Levy, S. et al. The diploid genome sequence of an individual human. PLoS Biol. 5, e254 (2007).

37. Ohno, S. Evolution By Gene Duplication (Springer-Verlag, New York, 1970).

38. Kim, M.Y. et al. Neonatal and adult CD4+CD3 - cells share similar gene expression profile, and neonatal cells up-regulate OX40 ligand in response to TL1A (TNFSF15). J. Immunol. 177, 3074-3081 (2006).

39. Fu, Y.X. \& Chaplin, D.D. Development and maturation of secondary Iymphoid tissues. Annu. Rev. Immunol. 17, 399-433 (1999).

40. Bekiaris, $V$. et al. Role of $\mathrm{CD} 30$ in $\mathrm{B} / \mathrm{T}$ segregation in the spleen. J. Immunol. 179, 7535-7543 (2007).

41. Bekiaris, V. et al. CD30 is required for CCL21 expression and CD4 T cell recruitment in the absence of lymphotoxin signals. J. Immunol. 182, 4771-4775 (2009).

42. Fu, Y.X. et al. Lymphotoxin-alpha (LTalpha) supports development of splenic follicular structure that is required for lgG responses. J. Exp. Med. 185, 2111-2120 (1997).

43. Kang, H.S. et al. Signaling via LTbetaR on the lamina propria stromal cells of the gut is required for IgA production. Nat. Immunol. 3, 576-582 (2002).

44. Muramatsu, M. et al. Class switch recombination and hypermutation require activation-induced cytidine deaminase (AID), a potential RNA editing enzyme [see comments]. Cell 102, 553-563 (2000).

45. Gong, Y.F., Xiang, L.X. \& Shao, J.Z. CD154-CD40 interactions are essential for thymus-dependent antibody production in zebrafish: insights into the origin of costimulatory pathway in helper T cell-regulated adaptive immunity in early vertebrates. J. Immunol. 182, 7749-7762 (2009).

46. Wilson, M. et al. What limits affinity maturation of antibodies in Xenopusthe rate of somatic mutation or the ability to select mutants? EMBO J. 11, 4337-4347 (1992).

47. Liu, Y.J., Zhang, J., Lane, P.J., Chan, E.Y. \& MacLennan, I.C. Sites of specific B cell activation in primary and secondary responses to T celldependent and T cell-independent antigens. Eur. J. Immunol. 21, 2951-2962 (1991).

48. Fu, Y.X., Huang, G., Wang, Y. \& Chaplin, D.D. Lymphotoxin-alphadependent spleen microenvironment supports the generation of memory $B$ cells and is required for their subsequent antigen-induced activation. J. Immunol. 164, 2508-2514 (2000).

49. Tsuji, M. et al. Requirement for lymphoid tissue-inducer cells in isolated follicle formation and $\mathrm{T}$ cell-independent immunoglobulin A generation in the gut. Immunity 29, 261-271 (2008).

50. Bengten, E. et al. Immunoglobulin isotypes: structure, function and genetics. In Origin And Evolution Of The Vertebrate Immune System (du Pasquier, L. and Litman, G.W., eds) 189-219 (Springer, Berlin, 2000).

51. Fagarasan, S. et al. Critical roles of activation-induced cytidine deaminase in the homeostasis of gut flora. Science 298, 1424-1427 (2002).

52. Peterson, D.A., McNulty, N.P., Guruge, J.L. \& Gordon, J.I. IgA response to symbiotic bacteria as a mediator of gut homeostasis. Cell Host Microbe 2, 328-339 (2007)
53. Bouskra, D. et al. Lymphoid tissue genesis induced by commensals through NOD1 regulates intestinal homeostasis. Nature 456, 507-510 (2008).

54. Croft, M. Co-stimulatory members of the TNFR family: keys to effective T-cell immunity? Nat. Rev. Immunol. 3, 609-620 (2003).

55. Watts, T.H. TNF/TNFR family members in costimulation of T cell responses. Annu. Rev. Immunol. 23, 23-68 (2005).

56. Gaspal, F.M. et al. Mice deficient in OX40 and CD30 signals lack memory antibody responses because of deficient CD4 T cell memory. J. Immunol. 174, 3891-3896 (2005).

57. Gaspal, F. et al. Critical synergy of CD30 and OX40 signals in CD4 T cell homeostasis and Th1 immunity to Salmonella. J. Immunol. 180, 2824-2829 (2008).

58. Rossi, S.W. et al. RANK signals from CD4(+)3(-) inducer cells regulate development of Aire-expressing epithelial cells in the thymic medulla. J. Exp. Med. 204, 1267-1272 (2007).

59. Anderson, G., Lane, P.J. \& Jenkinson, E.J. Generating intrathymic microenvironments to establish T-cell tolerance. Nat. Rev. Immunol. 7, 954-963 (2007).

60. Hikosaka, Y. et al. The cytokine RANKL produced by positively selected thymocytes fosters medullary thymic epithelial cells that express autoimmune regulator. Immunity 29, 438-450 (2008).

61. Guerau-de-Arellano, M., Martinic, M., Benoist, C. \& Mathis, D. Neonatal tolerance revisited: a perinatal window for Aire control of autoimmunity. J. Exp. Med. 206, 1245-1252 (2009).

62. Burnet, F.M. A modification of Jerne's theory of antibody production using the concept of clonal selection. Aust. J. Sci. 20, 67-69 (1957).

63. Aujla, S.J. et al. IL-22 mediates mucosal host defense against Gramnegative bacterial pneumonia. Nat. Med. 14, 275-281 (2008).

64. Zheng, Y. et al. Interleukin-22 mediates early host defense against attaching and effacing bacterial pathogens. Nat. Med. 14, 282-289 (2008).

65. Satoh-Takayama, N. et al. Microbial flora drives interleukin 22 production in intestinal NKp46+ cells that provide innate mucosal immune defense. Immunity 29, 958-970 (2008).

66. Cella, M. et al. A human natural killer cell subset provides an innate source of IL-22 for mucosal immunity. Nature 457, 722-725 (2009).

67. Sanos, S.L. et al. RORgammat and commensal microflora are required for the differentiation of mucosal interleukin 22-producing NKp46+ cells. Nat. Immunol. 10, 83-91 (2009).

68. Igawa, D., Sakai, M. \& Savan, R. An unexpected discovery of two interferon gamma-like genes along with interleukin (IL)-22 and -26 from teleost: IL-22 and -26 genes have been described for the first time outside mammals. Mol. Immunol. 43, 999-1009 (2006).

69. Lane, P.J. The architects of $\mathrm{B}$ and $\mathrm{T}$ cell immune responses. Immunity 29, 171-172 (2008). 